\title{
Optimal Transmission Expansion Planning Considering the Uncertainties of the Power Market
}

\author{
In-Su Bae*, Min-Kyun Son** and Jin-O Kim ${ }^{\dagger}$
}

\begin{abstract}
Today, as power trades between generators and loads are liberalized, the uncertainty level of power systems is rapidly increasing. Therefore, transmission operators are required to incorporate these uncertainties when establishing an investment plan for effective operation of transmission facilities. This paper proposes the methodology for an optimal solution of transmission expansion plans for the long-term in a deregulated power system. The proposed model uses the probabilistic cost of transmission congestion for various scenarios and the annual increasing rates of loads. The locations and the installation times of expanded transmissions lines with minimum cost are acquired by the model. To minimize the investment risk, the Mean-Variance Markowitz portfolio theory is applied to the model. In a case study, the optimal solution of a transmission expansion plan is obtained considering the uncertain power market.
\end{abstract}

Keywords: Optimal transmission expansion planning, Congestion cost, Markowitz, Monte Carlo simulation

\section{Nomenclature}

Expansion stage

Final expansion stage

Nodes

Scenario

Load duration factor

The set of node pairs where lines can be added

The set of all nodes

The set of all possible scenarios in the generation

Random variable number

Congestion cost $\$ / \mathrm{MWh}$ (penalty factor for congestion) for overloading power flow as $1 \mathrm{MW}$ (unit power) on the line at nodes $\mathrm{i}$ and $\mathrm{j}$

$\Delta P_{i j, k, l d, t} \quad$ The magnitude of overloading power flow on the line between nodes $i$ and $j$ in scenario $k$, load duration factor ld at the expansion stage $t$

$P_{i j, \max } \quad$ Transmission capacity for power flow on the line between nodes $\mathrm{i}$ and $\mathrm{j}$

$a \quad$ The positive integer weighted by the seriousness of overloading

$L D_{l d} \quad$ Load duration (hour) of load duration factor ld for a year

The probability of scenario $\mathrm{k}$ for the future

The number of transmission lines added between nodes $i$ and $\mathrm{j}$ at the expansion stage $\mathrm{t}$

$n_{i j, t}^{e x} \quad$ The number of existing transmission lines (include added lines) between nodes $i$ and $j$ at the expansion stage $t$

$t_{0} \quad$ The reference year for obtaining the present values of costs

I Annual discount rate

$c_{i j} \quad$ The cost per transmission line added between nodes $\mathrm{i}$ and $\mathrm{j}$

$o p_{i j} \quad$ The yearly operation cost of a transmission line between nodes $\mathrm{i}$ and $\mathrm{j}$

$\dagger$ Corresponding Author: Dept. of Electrical Engineering, Hanyang University, Korea. (jokim@hanyang.ac.kr)

* Dept. of Electrical Engineering, Kangwon National University, Korea. (isbae@kangwon.ac.kr)

** Dept. of Electrical Engineering, Hanyang University, Korea (gp22@hanyang.ac.kr)

Received: January 19, 2010; Accepted: March 23, 2010
$\mathrm{S}_{\mathrm{t}} \quad$ The node-branch incidence matrix at the expansion stage $\mathrm{t}$

$f_{k, l d, t} \quad$ The power flow vector at the expansion stage $t$ in scenario $\mathrm{k}$, load duration factor ld

$\mathrm{g}_{\mathrm{k}, \mathrm{ld}} \quad$ The generation vector in scenario $\mathrm{k}$, load duration factor ld

$\mathrm{d}_{\mathrm{k}, \mathrm{ld}} \quad$ The load vector in scenario $\mathrm{k}$, load duration factor ld

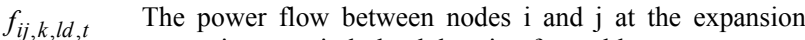
stage $\mathrm{t}$ in scenario $\mathrm{k}$, load duration factor ld

$\gamma_{i j} \quad$ The susceptance of a transmission line between nodes $\mathrm{i}$ and $\mathrm{j}$

$n_{i j, t}^{0} \quad$ The number of transmission lines between nodes $\mathrm{i}$ and $\mathrm{j}$ at $n_{i j, t} \quad$ the base case of the expansion stage $\mathrm{t}$

$\overline{n_{i j}} \quad$ The maximum number of transmission lines that can be $n_{i j} \quad$ added between nodes $\mathrm{i}$ and $\mathrm{j}$

$\theta_{i, k, l d, t} \quad$ The nodal angles at nodes $\mathrm{i}$ and $\mathrm{j}$ at the expansion stage $\mathrm{t}$ in $\theta_{j, k, l d, t} \quad$ scenario $\mathrm{k}$, load duration factor ld

$\mathrm{g}, \overline{\mathrm{g}} \quad$ Minimum, maximum generation level vector

$g_{h, k, l d} \quad$ Production quantity of generator $\mathrm{h}$ in scenario $\mathrm{k}$, load duration factor ld

$D_{l d} \quad$ Total production quantity of all generators (equal to total load quantity) in load duration factor ld

$\lambda_{h, k} \quad$ The participation factor of generator $\mathrm{h}$ for the pool market in scenario $\mathrm{k}$

$\beta \quad$ Weighting factor for minimizing the variance of congestion cost in the optimization

$\sigma_{l d} \quad$ The standard deviation of hourly congestion cost in load duration factor ld

\section{Introduction}

In the environment of a deregulated power market, market participants in power systems compete with others and try to adopt strategies for maximizing their profits.

As a result of cases originating from the uncertainty of generation and load quantities with increased usage rates for the facilities, it is difficult for power system planners to design cost-effective and reliable power systems.

In particular, transmission planners should fulfill the minimum cost investment plan with the maximum effect of 
the operation for the transmission facilities.

Poor investment planning for the transmission expansion of power systems usually gives rise to congestion costs. Congestion is primarily the obstacle for the effectiveness of the power system and reduces the fairness level for opportunities of market participants. Therefore, it is essential for transmission planners to try to establish investment plans to minimize the congestion cost and level [1].

As transmission investment has a high cost and entails a complicated process, cost-effective investment planning has been determined for future cases, such as power flow patterns from the production and consumption of generation and loads. Therefore, transmission planners are required to forecast the amount of generation and loads on a time basis.

To improve transmission investment, this paper proposes a methodology for optimal investment planning that considers the uncertainty problem of future power systems. In this paper, an optimization model is suggested to minimize the sum of transmission expansion, operation and congestion costs, and the decision variables of the model are the locations and times for the expanded transmission lines. To consider the uncertainty factors of the future for long-term investment, the congestion cost is obtained in this paper with the probabilistic method and the actions of the generators are analyzed by the probabilities based on the scenarios. In addition, the annual increasing rates of the load quantities are treated as random variables with the probability distribution. To minimize the investment risk, the Mean-Variance Markowitz portfolio theory is newly applied to the optimization model for a transmission expansion plan.

\section{Mathematical Formulation for the Minimum Cost Model}

\subsection{Probabilistic Congestion Cost}

The congestion cost of a transmission system happens by a constrained quantity of power flow on the capacity of transmission lines. It is related to the magnitude of overloading power on the lines where the power flows of the lines are influenced by the actions of generation and loads. For transmission expansion planning, the cost of unit overloading power (MW) is an important factor and it is essential to estimate the overloading levels of the lines. Hourly Congestion Cost $H C C_{i j, k, l d, t}$ of a transmission line between nodes $i$ and $j$ can be represented as Eq. (1) [2]

$$
H C C_{i j, k, l d, t}=\alpha_{i j} \Delta P_{i j, k, l d, t}\left(\frac{\Delta P_{i j, k, l d, t}}{P_{i j, \max }}+1\right)^{a}
$$

in scenario $k$ with load duration factor $l d$ at the expansion stage $t$. The term $\left(\frac{\Delta P_{i j, k, l d, t}}{P_{i j, \max }}+1\right)^{a}$ indicates the relative overloading level.
The load is continually varied for a year and the changes in load magnitudes of the regions or nodes leads to several patterns of power flow and influence the congestion cost of the transmission system. In this paper, to consider the changeable loads, load duration for a year is classified into several sections based on the change patterns in loads by time. Hourly congestion cost is weighted by load durations $L D_{l d}$ for sections $l d$ and Yearly Congestion Cost $Y C C_{i j, k, t}$ can be obtained as

$$
Y C C_{i j, k, t}=\sum_{l d} L D_{l d}\left(H C C_{i j, k, l d, t}\right)
$$

As can be seen in Eq. (1), the changeable power flow patterns have influence on the congestion cost of the transmission system, and therefore, random factors must be considered for various cases to obtain the congestion cost in the market with so many uncertainty factors. In this paper, the expected value of the congestion cost in various scenarios is included in the optimization model as the performance factor of congestion. The expected value of the congestion cost $E\left\{Y C C_{i j, k, t}\right\}$ for all possible scenarios is presented as

$$
E\left\{Y C C_{i j, k, t}\right\}=\sum_{k} \operatorname{pro}_{k}\left(Y C C_{i j, k, t}\right)
$$

\subsection{Obtaining the Present Value of Cost}

The planning horizon for the long-term operation of transmission can be divided into several periods by expansion times. Because the times when the expansion, operation and congestion costs occur for the planning horizon have influence on the present value of expenses based on the reference year, times of expenses related to transmission should be considered to evaluate the plans. Accordingly, all expenses for the planning horizon are transformed into the present value for the reference year.

If it is assumed that the lines are expanded periodically, the stage of expansion is represented by the integer index $t$, where the expansion years are $y_{t}, t=0, \cdots, T$. If the number of added lines between nodes $i$ and $j$ at expansion stage $t$ is given as $n_{i j, t}$, the number of existing lines $n_{i j, t}^{e x}$ is increased by $n_{i j, t}$ and the relationship between these two variables can be expressed as

$$
n_{i j, t}^{e x}=n_{i j, 0}+\sum_{q=1}^{t} n_{i j, q}
$$

where $n_{i j, 0}$ is the number of lines at the reference year.

The investment cost $c\left(n_{i j, t}\right)$ at expansion stage $t$ is the function of the number of added lines $n_{i j, t}$, while the yearly operation and congestion cost $d\left(n_{i j, t}^{e x}\right)$ is of the number of existing lines $n_{i j, t}^{e x}$.

Each cost can be transformed as the present values on 
the reference year $y_{0}$. The present values of the total investment $\operatorname{cost} c_{\text {total }}\left(n_{i j, t}\right)$ and yearly operation and congestion cost $d_{\text {total }}\left(n_{i j, t}^{\text {ex }}\right)$ on the reference year are given in Eqs.

(5) and (6), respectively [3].

$$
\begin{aligned}
& c_{\text {total }}\left(n_{i j, t}\right)=(1-I)^{y_{1}-y_{0}} c\left(n_{i j, 1}\right)+(1-I)^{y_{2}-y_{0}} c\left(n_{i j, 2}\right)+ \\
& \cdots+(1-I)^{y_{t}-y_{0}} c\left(n_{i j, t}\right)+\cdots+(1-I)^{y_{T}-y_{0}} c\left(n_{i j, T}\right) \\
& d_{\text {total }}\left(n_{i j, t}^{e x}\right)=\sum_{r=y_{1}}^{y_{2}-1}(1-I)^{r-y_{0}} d\left(n_{i j, 1}^{e x}\right)+\sum_{r=y_{2}}^{y_{3}-1}(1-I)^{r-y_{0}} d\left(n_{i j, 2}^{e x}\right)+ \\
& \cdots+\sum_{r=y_{t}}^{y_{t+1}-1}(1-I)^{r-y_{0}} d\left(n_{i j, t}^{e x}\right)+\cdots+\sum_{r=y_{T}}^{y_{T+1}-1}(1-I)^{r-y_{0}} d\left(n_{i j, T}^{e x}\right)
\end{aligned}
$$

The yearly cost for operation and congestion can be generally assumed to be uniform up to the time of new investment for expansion and changed at the time of new investment.

For convenience, the coefficients $\delta_{i n v, t}$ and $\delta_{a n n, t}$ are defined as

$$
\begin{gathered}
\delta_{i n v, t}=(1-I)^{y_{t}-y_{0}} \\
\delta_{a n n, t}=\sum_{p=y_{t}-y_{0}}^{y_{t+1}-1-y_{0}}(1-I)^{p}
\end{gathered}
$$

where $\delta_{i n v, t}$ is the discount factor to obtain the present value of investment cost for transmission expansion at the expansion stage $t$, and $\delta_{a n n, t}$ is the discount factor related to yearly cost which is the summation of every year during the period between the investment for the expansion

Then, Eqs. (5) and (6) are expressed simply as follows:

$$
\begin{aligned}
& c_{\text {total }}\left(n_{i j, t}\right)=\delta_{i n v, 1} c\left(n_{i j, 1}\right)+\delta_{i n v, 2} c\left(n_{i j, 2}\right)+\cdots+\delta_{i n v, t} c\left(n_{i j, t}\right)+ \\
& \cdots+\delta_{i n v, T} c\left(n_{i j, T}\right)=\sum_{t=1}^{T} \delta_{i n v, t} c\left(n_{i j, t}\right) \\
& d_{t o t a l}\left(n_{i j, t}^{e x}\right)=\delta_{a m n, 1} d\left(n_{i j, 1}^{e x}\right)+\delta_{a n n, 2} d\left(n_{i j, 2}^{e x}\right)+\cdots+\delta_{a n n, t} d\left(n_{i j, t}^{e x}\right)+ \\
& \cdots+\delta_{a m n, T} d\left(n_{i j, T}^{e x}\right)=\sum_{t=1}^{T} \delta_{a n n, t} d\left(n_{i j, t}^{e x}\right)
\end{aligned}
$$

\subsection{Investment and Operation Cost}

In this paper, an optimization model is proposed to minimize the cost by long-term transmission expansion planning. The optimization model consists of the costs for the transmission expansion, operation and congestion for the planning horizon.

The investment cost for expansion $c\left(n_{i j, t}\right)$ can be expressed simply by $c_{i j} n_{i j, t}$ and is transformed to the present value as Eq. (11),

$$
I C(t)=\delta_{i n v, t}\left(\sum_{(i, j) \in x p} c_{i j} n_{i j, t}\right)
$$

where $(i, j) \in x p$ denotes the location of the lines to be expanded.

The operation cost for existing lines can be expressed simply by $o p_{i j} n_{i j, t}^{e x}$ and is transformed to the present value as

$$
O C(t)=\delta_{a n n, t}\left(\sum_{(i, j) \in \text { all }} o p_{i j} n_{i j, t}^{e x}\right)
$$

where $(i, j) \in$ all denotes the location of the all lines.

\subsection{Congestion Cost}

As explained previously in Eq. (3), the expected value of the congestion cost for existing lines is used for optimization, and is also transformed to the present value as Eq. (13),

$$
C C(t)=\delta_{\text {ann }, t}\left(\sum_{(i, j) \in \text { all }} E\left\{Y C C_{i j, k, t}\right\}\right)
$$

This paper assumes that the load is shared by all generators participating in the market. The participation factors of the generators are the constants which represent the market share of the production quantities of the corresponding generators as Eqs. (14) and (15) [4], [5].

$$
\begin{gathered}
g_{h, k, l d}=\lambda_{h, k} D_{l d}, \forall k, l d \\
\sum_{h} \lambda_{h, k}=1, \forall k \\
h \in N
\end{gathered}
$$

where $\lambda_{h, k}$ represents the participation factor of generator $h$ in scenario $k$ and it reflects the generation level $g_{h, k, l d}$ of generator $h$. The value of $\lambda_{h, k}$ is in the range of 0 to 1 and indicates the level of generator $h$ for undertaking the part of total load quantity assumed to be 1 .

For long-term transmission planning, it is required to analyze the change of future forecasted loads. By identifying past load data, the yearly change in load can be predicted but have uncertainties or are estimated values with some errors. The annual increasing or decreasing rates of load quantities are used as the indices of the change in this paper, and the yearly increasing rates with the uncertainties for each node are treated as random variables of a probability distribution.

In this paper, the probability distribution for the annual increasing rate is assumed to be the normal distribution as Eq. (17) [6].

$$
f\left(x_{r, h}\right)=\frac{1}{\sigma_{h} \sqrt{2 \pi}} \exp \left[-\frac{\left(x_{r, h}-\mu_{h}\right)^{2}}{2 \sigma_{h}^{2}}\right]
$$

where $\mu_{h}$ and $\sigma_{h}$ are the mean and standard deviation for the increasing rate of load quantity at load bus $h$, respectively. 
To obtain the total expected congestion cost with the uncertainty of the increasing rates of loads, a Monte Carlo Simulation is applied as shown in Fig. 1.

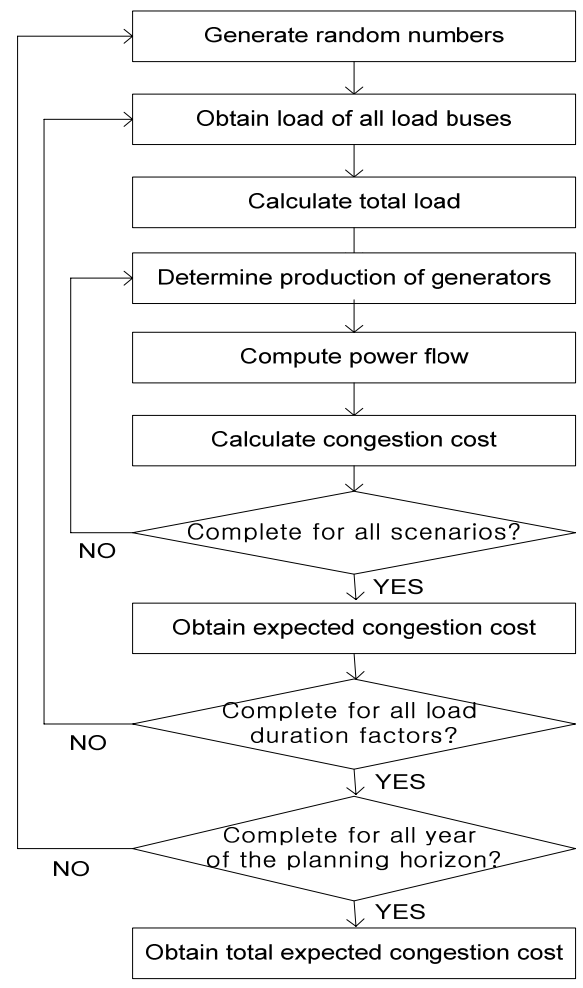

Fig. 1. Flow chart for the simulation.

\subsection{The Optimization Model}

Finally, the proposed optimization model is given as Eq. (18) to evaluate the investment plan of transmission expansion for the long-term,

$$
\operatorname{Minimize} \sum_{t=1}^{T}\{I C(t)+O C(t)+C C(t)\}
$$

Subject to

$$
\begin{gathered}
\mathrm{S}_{\mathrm{t}} \mathrm{f}_{\mathrm{k}, \mathrm{ld}, \mathrm{t}}+\mathrm{g}_{\mathrm{k}, \mathrm{ld}}=\mathrm{d}_{\mathrm{k}, \mathrm{ld}}, \forall \mathrm{k}, \mathrm{ld}, \mathrm{t} \\
f_{i j, k, l d, t}-\gamma_{i j}\left(n_{i j, t}^{0}+n_{i j, t}\right)\left(\theta_{i, k, l d, t}-\theta_{j, k, l d, t}\right)=0, \forall k, l d, t \\
\mathrm{~g} \leq \mathrm{g}_{\mathrm{k}, \mathrm{ld}} \leq \overline{\mathrm{g}}, \forall \mathrm{k}, \mathrm{ld} \\
0 \leq n_{i j, t} \leq \bar{n}_{i j}, \forall t \\
\Omega \subset N \\
i, j \subset \Omega, k \in K
\end{gathered}
$$

where $I C(t), O C(t)$ and $C C(t)$ are given in Eqs. (11), (12) and (13). The constraints are power balance, DC power flow, production limit of the generators and the number of added transmission lines which are represented by Eqs. (19) - (24). The decision variable of the model is $n_{i j, t}$, which is the location and the number of expanded lines between nodes $i$ and $j$ at the expansion stage $t$.

\section{Minimizing the Risk of Congestion Cost}

Markowitz suggested the portfolio theory for the minimum variance of profit related to the expected profit as an investment strategy [7]. The portfolio theory considers the variances of expected return and cost as an investment risk, and using this theory, the variance can be minimized as a risk. By this theory, standard deviation with a penalty factor is added to the objective function for minimizing the cost as Eq. (25).

$$
\text { Minimize } f(x)+E\{g(x)\}+\beta \sqrt{\operatorname{Var}\{g(x)}\}
$$

where $f(x)$ is the deterministic cost without a probability factor for the investment decision variable $x$, and $E\{g(x)\}$ and $\sqrt{\operatorname{Var}\{g(x)\}}$ indicate the expected value and the standard deviation of the cost with probability factors. $\beta$ is the penalty factor for minimizing $\sqrt{\operatorname{Var}\{g(x)\}}$ as an investment risk. The value of $\beta$ can be chosen by one who makes a decision and is weighted by the importance of minimizing the variance of cost in the optimization process.

In the optimization model, the Markowitz portfolio theory can be applied to the concept of investment risk by the variance of the congestion cost with probabilistic factors. The costs of investment and operation for the transmission expansion can be considered as the deterministic function of $f(x)$ and the congestion cost is related to $g(x)$ due to the probability factor of scenarios for the congestion cost. Investment decision $x$ is also equivalent to the number of added lines $n_{i j, t}$. Generally, the lower the variance of the congestion cost, the less risky the strategy of investment.

For convenience, if a new variable is defined for the present value of the hourly congestion cost as follows:

$$
z_{k, l d}=\sum_{t=1}^{T} \delta_{a n n, t}\left(H C C_{i j, k, l d, t}\right)
$$

then the standard deviation of the hourly congestion cost for the load duration factor $l d$ is represented in Eq. (27).

$$
\sigma_{l d}=\sqrt{E\left\{z_{k . l d}^{2}\right\}-E^{2}\left\{z_{k, l d}\right\}}
$$

As shown in Eq. (28), the variance of congestion cost and the penalty factor $\beta$ are added to the original optimization model in Eq. (14) in order to find a less risky solution.

$$
\operatorname{Minimize} \sum_{t=1}^{T}\{I C(t)+O C(t)+C C(t)\}+\beta \sum_{l d} L D_{d} \sigma_{l d}
$$

where the load duration $L D_{l d}$ of the factor $l d$ is weighted to the standard deviation of the congestion cost to consider the whole year. 


\section{Case Study}

In this paper, the IEEE Reliability Test System with 24 buses as shown in Fig. 2 was used for the case study [8]. In the case study, the planning horizon for transmission expansion is assumed to be 10 years and investment for expansion is performed by periods of five years for simple calculation. Therefore, the transmission system is expanded twice in years 0 and 5 in order to analyze the total cost and

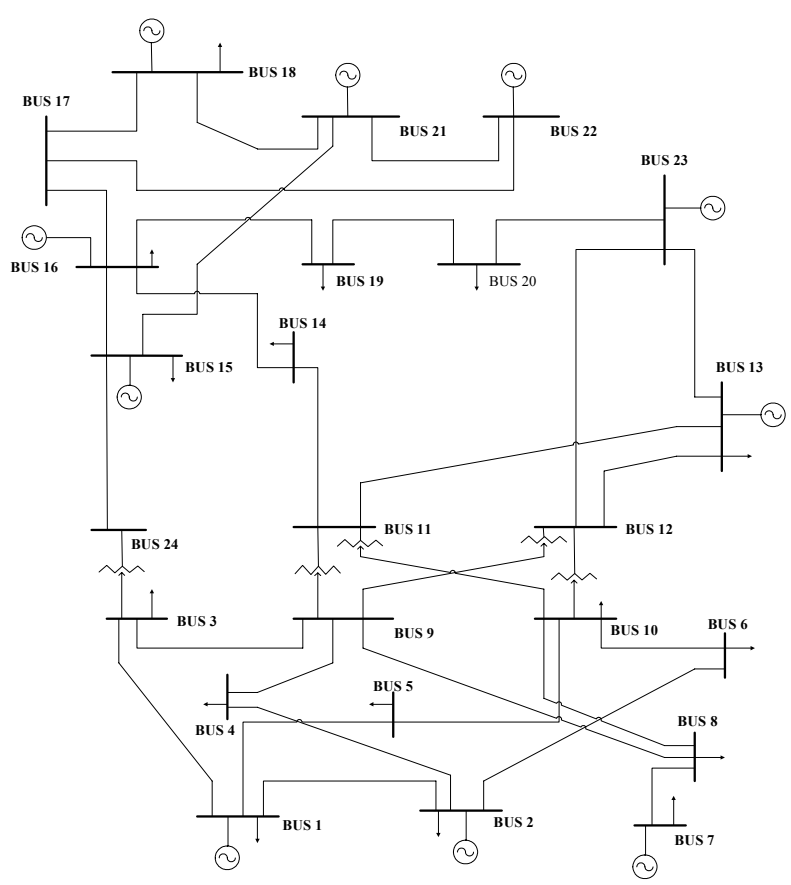

Fig. 2. IEEE Reliability Test System with 24 buses. obtain optimal planning for the 10 year period. The annual discount rate $I$ is assumed to be 0.1 and the penalty factor for congestion $\alpha_{i j}$ in Eq. (1) is equal to $50 \$ / \mathrm{MWh}$ in the case study. The overloading weighting factor $a$ is assumed to be 1.5 .

The case in generations consists of five scenarios and their occurrence probabilities are tabulated in Table 1.

The term of the one year load duration is divided into two periods, Off-Peak and Peak and their load duration factors are assumed in Table 2.

The participation factors of all generators connected to the 10 generation buses are also tabulated in Table 3 for the five scenarios. Table 4 informs the load data of the IEEE RTS System with Peak and Off-Peak sections for the year zero. The mean and standard deviation of the increasing rates of load quantities are also assumed for the first and second five years, respectively.

Table 1. Occurrence probabilities of the scenarios

\begin{tabular}{c|c|c|c|c|c}
\hline $\mathrm{k}$ & $\begin{array}{c}\text { scenario } \\
1\end{array}$ & $\begin{array}{c}\text { scenario } \\
2\end{array}$ & $\begin{array}{c}\text { scenario } \\
3\end{array}$ & $\begin{array}{c}\text { scenario } \\
4\end{array}$ & $\begin{array}{c}\text { scenario } \\
5\end{array}$ \\
\hline pro $_{k}$ & 0.1 & 0.2 & 0.4 & 0.2 & 0.1 \\
\hline
\end{tabular}

Table 2. The classification of load duration factors

\begin{tabular}{c|c}
\hline & Term of $L D_{l d}$ (hour) \\
\hline Off-Peak $(l d=1)$ & 4871 \\
\hline Peak $(l d=2)$ & 3889 \\
\hline One Year & 8760 \\
\hline
\end{tabular}

\subsection{Optimal Transmission Expansion Plan}

Tables 5 and 6 present the expansion plan for the minimal total cost for the 10 years as the planning horizon. As

Table 3. Five scenarios with participation factors of generators

\begin{tabular}{c|c|c|c|c|c|c|c|c|c|c}
\hline node & 1 & 2 & 7 & 13 & 15 & 16 & 18 & 21 & 22 & 23 \\
\hline scenario 1 & 0.056 & 0.056 & 0.079 & 0.095 & 0.070 & 0.053 & 0.133 & 0.137 & 0.095 & 0.226 \\
\hline scenario 2 & 0.056 & 0.056 & 0.079 & 0.095 & 0.080 & 0.063 & 0.113 & 0.117 & 0.105 & 0.236 \\
\hline scenario 3 & 0.056 & 0.056 & 0.079 & 0.085 & 0.060 & 0.043 & 0.143 & 0.147 & 0.095 & 0.236 \\
\hline scenario 4 & 0.056 & 0.056 & 0.079 & 0.105 & 0.070 & 0.053 & 0.133 & 0.137 & 0.105 & 0.206 \\
\hline scenario 5 & 0.046 & 0.046 & 0.069 & 0.105 & 0.080 & 0.063 & 0.133 & 0.137 & 0.095 & 0.226 \\
\hline
\end{tabular}

Table 4. Load data of IEEE RTS system

\begin{tabular}{|c|c|c|c|c|c|c|c|c|c|c|c|c|c|c|c|c|c|}
\hline Node & 1 & 2 & 3 & 4 & 5 & 6 & 7 & 8 & 9 & 10 & 13 & 14 & 15 & 16 & 18 & 19 & 20 \\
\hline \multicolumn{18}{|c|}{ The base loads (zero year) MWh } \\
\hline $\begin{array}{l}\text { Off- } \\
\text { Peak }\end{array}$ & 108 & 97 & 180 & 74 & 71 & 136 & 125 & 171 & 175 & 195 & 265 & 194 & 317 & 100 & 333 & 181 & 128 \\
\hline Peak & 140 & 126 & 234 & 96 & 92 & 177 & 163 & 222 & 228 & 254 & 345 & 252 & 412 & 130 & 433 & 235 & 186 \\
\hline \multicolumn{18}{|c|}{ The annual increasing rates of loads between 0 year and 5 year (S.D. : Standard Deviation) } \\
\hline MEAN & 0.051 & 0.131 & 0.051 & 0.051 & 0.131 & 0.051 & 0.131 & 0.051 & 0.131 & 0.051 & 0.051 & 0.051 & 0.051 & 0.051 & 0.051 & 0.131 & 0.051 \\
\hline S.D. & 0.065 & 0.070 & 0.065 & 0.021 & 0.021 & 0.065 & 0.070 & 0.065 & 0.070 & 0.065 & 0.065 & 0.065 & 0.065 & 0.021 & 0.065 & 0.070 & 0.021 \\
\hline \multicolumn{18}{|c|}{ The annual increasing rates of loads between 5 year and 10 year } \\
\hline MEAN & 0.052 & 0.080 & 0.052 & 0.060 & 0.060 & 0.052 & 0.080 & 0.052 & 0.080 & 0.052 & 0.052 & 0.052 & 0.052 & 0.060 & 0.052 & 0.080 & 0.060 \\
\hline S.D. & 0.008 & 0.018 & 0.008 & 0.021 & 0.021 & 0.008 & 0.018 & 0.008 & 0.018 & 0.008 & 0.008 & 0.008 & 0.008 & 0.021 & 0.008 & 0.018 & 0.021 \\
\hline
\end{tabular}


the load quantities of the load buses increase year by year for the planning horizon, the transmission system gets more and more congested and the degree of congestion for years 6-10 is more significant than that for years 1-5. For this reason, the number of added lines in year 5 is revealed to be larger than that in year 0 in the case study.

The cost of the optimal plan is shown in Table 7. In this table, it is expected that if the expansion cost is increased, the congestion cost would decrease while operation costs would increase, and the resulting total cost would increase more or less compared to that of the optimal result. In contrast, for the case with a lower expansion cost, vice versa, and the result would also be increased.

Table 5. The number of added transmission lines in year 0

\begin{tabular}{c|c|c|c|c|c|c}
\hline From-To & $3-24$ & $6-10$ & $9-12$ & $10-12$ & $12-23$ & $13-23$ \\
\hline Number & 1 & 1 & 1 & 2 & 3 & 1 \\
\hline
\end{tabular}

Table 6. Number of added transmission lines in year 5

\begin{tabular}{c|c|c|c|c|c|c}
\hline From-To & $3-24$ & $6-10$ & $14-16$ & $15-21$ & $16-17$ & $17-18$ \\
\hline Number & 1 & 1 & 1 & 2 & 3 & 1 \\
\hline
\end{tabular}

\begin{tabular}{c|c|c|c|c|c|c}
\hline From-To & $14-16$ & $15-21$ & $15-24$ & $16-17$ & $16-19$ & $17-18$ \\
\hline Number & 1 & 1 & 1 & 2 & 3 & 1 \\
\hline
\end{tabular}

Table 7. Optimal result for 10 years

\begin{tabular}{c|c|c|c}
\hline Expansion Cost & Operation Cost & Congestion Cost & Total Cost \\
\hline $1.5697 \times 10^{7}$ & $3.3442 \times 10^{6}$ & $8.7120 \times 10^{6}$ & $2.7753 \times 10^{7}$ \\
\hline
\end{tabular}

\subsection{Optimal Transmission Expansion Plan with Penalty Factor of Risk}

To analyze the risk effect, the Markowitz portfolio theory was applied, and the number of added lines at nodes 10-12 was increased from two to three. Except for the added lines at nodes 10-12, the optimal solution with the penalty factor was identical to those presented in Tables 5 and 6 .

In Table 8 , with the penalty factor $\beta$ equal to 1.5 , the acquired standard deviation of the congestion cost is lower than that of the case without factor, while the total cost is slightly increased. The solution with a lower variance of the congestion cost can be analyzed to be a less risky investment plan. However, there is a trade-off between the risk and investment. The action for choosing an optimal plan with or without the penalty factor is determined by the characteristics of the decision maker. In cases where the transmission planner is risk averse as minimizing the variance of the congestion cost, the optimal plan by the penalty factor is chosen. On the contrary, the transmission planner who prefers greater risk has a focus on minimizing only total cost without the penalty factor.
Table 8. Optimal result with and without the penalty factor of standard deviation

\begin{tabular}{c|c|c}
\hline & Without Penalty $(\beta=0)$ & With Penalty $(\beta=1.5)$ \\
\hline Total Cost & $2.7753 \times 107$ & $2.7947 \times 107$ \\
\hline $\begin{array}{c}\text { Standard deviation of } \\
\text { Congestion Cost }\end{array}$ & $5.6332 \times 106$ & $5.5027 \times 106$ \\
\hline
\end{tabular}

\section{Conclusion}

As power trades between producers and consumers have recently been deregulated, the conditions for the operation of a power system are becoming increasingly uncertain. In this power system environment, a deliberate investment plan for transmission systems must be made taking into consideration the forecasted situation of power systems by a transmission planner. To aid this decision-making process, this paper proposes the probabilistic methodology for optimal transmission expansion under uncertain power market conditions.

In this paper, increasing rates of load quantities and congestion cost are considered as a probability distribution. An optimal solution including the locations and the number of added transmission lines is proposed to minimize cost under conditions of future market uncertainty. In addition, the Markowitz theory is introduced to the proposed model, and investment risk is considered. In a case study, the proposed method is verified as an effective method for obtaining an optimal transmission expansion plan in a deregulated power system.

\section{References}

[1] $\mathrm{H}$. He and $\mathrm{Z} \mathrm{Xu}$, "Transmission congestion and its social effects," IEEE Power Engineering Society General Meeting, Vol. 1, pp. 350-354, June 2005.

[2] Xiao Hongfei, Li Weidong and Wei Liming, "Congestion assessment for transmission planning in competitive electricity markets," IEEE/PES Transmission and Distribution Conference \& Exhibition: Asia and Pacific, Dalian, China, 2005.

[3] A. H. Escobar, R. A. Gallego and R. Romero, "Multistage and coordinated planning of the expansion of transmission systems," IEEE Transactions on Power Systems, Vol. 19, No. 2, pp. 735-744, May 2004.

[4] Daniel S. Kirschen and Goran Strbac, Fundamentals of Power System Economics, John Wiley \& Sons, 2004.

[5] R. Fang and D. J. Hill, "A new strategy for transmission expansion in competitive electricity markets," IEEE Transactions on Power Systems, Vol. 18, No. 4, pp. 374-380, Feb. 2003.

[6] R. Rillinton and R. N. Allan, Reliability Evaluating of Engineering System, 2nd Edition, Plenum Press, New York, 1996. 
[7] B. H. Kang, The theory of investment, Pakyoungsa, 2004.

[8] "IEEE reliability test system-1996," IEEE Transactions on Power Systems, Vol. 14, No. 3, pp. 10101020, Aug. 1999.

In-Su Bae received his B.S., M.S. and Ph.D. degrees from Hanyang University, Seoul, Korea, in 1998, 2003 and 2007, respectively. Currently, he is an Instructor with the Department of Electrical Engineering at Kangwon National University, Samcheok, Korea.

Min-Kyun Son received his B.S. and M.S. degrees from the Department of Electrical Engineering at Hanyang University. His research interest is transmission expansion planning.
Jin-O Kim received his B.S. and M.S. degrees in electrical engineering from Seoul National University, Seoul, Korea, in 1980 and 1983, respectively, and his Ph.D. degree from Texas A\&M University in 1991. Currently, he is a Professor with the Department of Electrical Engineering at Hanyang University, Seoul, Korea. 